\title{
Influencia del agente cementante y el envejecimiento en el color de carillas cerámicas, evaluación ex vivo
}

\author{
Influence of the cementing agent and aging in the color of ceramic veneers, ex vivo \\ evaluation
}

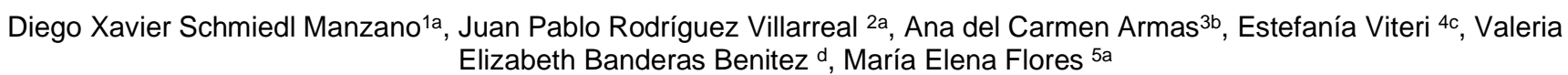

\section{RESUMEN}

Objetivo: Las carillas de cerámica se muestran como una excelente alternativa para mejorar la estética dental con propiedades ópticas y mecánicas ideales. El objetivo de este estudio fue evaluar la influencia del agente cementante y el envejecimiento artificial sobre el color. Materiales y métodos: Se utilizaron 30 premolares humanos los cuales fueron tallados para recibir carillas, confeccionadas con porcelana de $0,5 \mathrm{~mm}$ de grosor, de un mismo color. Los conjuntos diente-carilla fueron separados aleatoriamente en tres grupos $(n=10)$, una primera medición de color fue ejecutada sobre cada diente mediante colorímetro Vita Easyshade. El proceso de cementación fue realizado según el grupo, con cemento RelyX ultimate, RelyX Veneer y resina fluida Tetric-N-Flow, realizándose la medición de color; tras el desafío de envejecimiento, los conjuntos fueron sometidos a una nueva evaluación de color. Los datos obtenidos de las mediciones fueron analizadas mediante ANOVA con GamesHowell (software SPSS 21). Resultados: Se encontró menor variación entre los periodos evaluados con el uso del cemento RelyX ultimate $(p=0,384)$. Conclusiones: El envejecimiento artificial provocó cambio de color en todos los materiales evaluados. El cemento RelyX Veneer y la resina fluida Tetric-N-Flow presentaron mayor inestabilidad con rangos de color clínicamente aceptables.

PALABRAS CLAVE: Carillas; Color; Cementos de Resina. (Fuente: DeCS BIREME)

\begin{abstract}
Objetive: Ceramic veneers are shown as an excellent alternative to improve dental aesthetics with ideal optical and mechanical properties. The objective of this study, was to evaluate the influence of the cementing agent and artificial aging on the veneer's color. Material and Methods: For this, 30 human premolars were carved to receive veneers made with $0.5 \mathrm{~mm}$ thick porcelain of the same color. The tooth-veneer sets were randomly separated into three groups $(n=10)$. A first color measurement was performed on each tooth using the Vita Easyshade colorimeter. The cementing process was carried out according to the group, with RelyX ultimate cement, RelyX Veneer and Tetric-N-Flow fluid resin; performing the color measurement. After heat aging treatment, the sets were subjected to a new color evaluation. The data obtained from the measurements, were analyzed by ANOVA with Games - Howell (software SPSS 21). Results: Less variation was found between the periods evaluated with the use of RelyX ultimate cement $(p=0.384)$. Conclusions: Artificial aging caused a color change in all materials evaluated. RelyX Veneer cement and Tetric-N-Flow fluid resin showed greater instability with clinically acceptable color ranges.
\end{abstract}

KEY WORDS: Veneers; Color; Resin Cements. (Source: MeSH NLM)

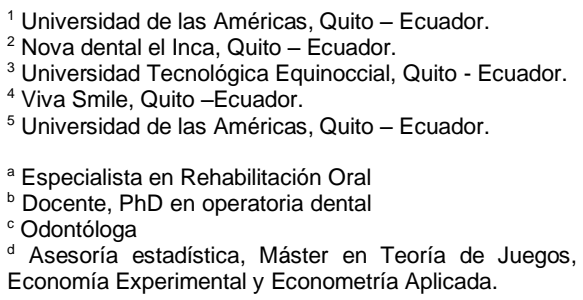

\section{Correspondencia:}

Juan Pablo Rodríguez Villarreal

Humberto Albornoz y Barros de San Millan OE 6 - 155. Quito, Ecuador. Celular: +593982036238

Correo electrónico: pablorodri-@hotmail.es

Citar como: Schmiedl D. Rodriguez JP. Arma A. Viteri E. Banderas V. Flores M. Influencia del agente cementante y el envejecimiento en el color de carillas cerámicas, evaluación ex vivo. KIRU. 2020; 17(1): 5 - 9. https://doi.org/10.24265/kiru.2020.v17n1.01 


\section{INTRODUCCIÓN}

Las carillas de cerámica constituyen la elección mínimamente invasiva, más adecuada para armonizar la sonrisa en dientes anteriores (1), debido a sus propiedades ópticas y mecánicas, otorgando una apariencia natural al diente ${ }^{(2)}$. Su uso está indicado para cerrar diastemas, restaurar defectos morfológicos, estructurales y funcionales, tratar dientes fracturados o ligeramente desalineados $y$ decolorados ${ }^{(3)}$. Su grosor generalmente entre 0,3 a 1,5 $\mathrm{mm}{ }^{(4)}$, puede verse influenciado por el agente cementante empleado, el cual puede inducir cambios en la coloración final del tratamiento, donde mientras más delgado es el grosor de la restauración, mayores son los cambios de color (5), atribuidos a las aminas terciarias presentes en el agente cementante (6). Notando mayor estabilidad con el uso de cementos del tipo dual (7). Pese a esto, los cementos de fotopolimerización muestran atributos interesantes que requieren ser considerados, de ahí que este estudio busca evaluar la estabilidad del color post cementación, utilizando tres materiales luego de ser sometidos a un desafío térmico de envejecimiento.

\section{MATERIAL Y MÉTODOS}

Se plantea un estudio in vitro, en el cual 30 premolares inferiores humanos sanos, obtenidos mediante carta de donación, extraídos por indicación terapéutica y tras eliminación de restos de tejido blando mediante cureta Gracey 13-14 (Bader, España) y hoja de bisturí \#15, fueron lavados con polvo de piedra pómez y cepillo profiláctico a baja velocidad, almacenados en suero fisiológico bajo refrigeración hasta su empleo y adaptados en un molde de acrílico (VeracrylColombia) dejando expuesta su corona dental para facilitar su manipulación.

Las superficies vestibulares de cada diente fueron registradas mediante una matriz de silicona por condensación (Zetaplus Zhermack, Zhermack SpaItaly) elaborada como herramienta para controlar el desgaste, tras lo cual las superficies fueron preparadas de forma estandarizada empleando puntas diamantadas grano medio 848R (Jota Swiz madeSuiza) en alta velocidad (NSK PAP-SU B2, NSKJapón) y buena refrigeración, siguiendo las vertientes vestibulares con una profundidad de desgaste de 0,5 $\mathrm{mm}$ controlado mediante sonda periodontal (HiuFriedy USA).

Concluida la preparación, fue ejecutada una impresión con silicona de adición (Helite HD Zhermack, Zhermack Spa-Italy), el modelo obtenido con el vaciado fue troquelado y se enceró sobre él una carilla con cera para modelado amarilla (Yeti Dental Produkte, Engen, Germany). Utilizando la técnica de "la cera perdida" e inyectando un bloque de cerámica
(IPS Empress Esthetic, Ivoclar Vivadent, Schaan, Leichtenstein) de color A2 de mediana translucidez, se obtuvo una carilla cerámica, la cual, tras su pulido, fue adaptada en cada una de las preparaciones y estandarizada en su espesor mediante calibrador electrónico (Mitutoyo Corporation, Tokyo, Japón). En este momento, se realizó una primera evaluación de color mediante colorímetro Vita Easyshade (VitazahnFabrik-Alemania), colocando la punta lo más cerca posible de la superficie vestibular intacta, con la superficie húmeda y bajo luz natural.

Los conjuntos "diente y carilla" fueron divididos aleatoriamente en tres grupos $(n=10)$. Las superficies vestibulares de los dientes fueron lavadas con polvo de piedra pómez y agua con un cepillo profiláctico en baja velocidad, tras el secado se procedió a colocar ácido ortofosfórico al $37 \%$ (FGM-Brasil) durante 30 segundos, se lavó por 60 segundos y se secó utilizando papel absorbente. A continuación, se aplicaron dos finas capas de sistema adhesivo (One Coat, FGM-Brazil) mediante técnica activa durante 10 segundos, aplicando aire entre las capas para volatilizar el vehículo y fotopolimerizando por 20 segundos con lámpara LED a intensidad de $480 \mathrm{~nm}$ y potencia de $1500 \mathrm{~mW} / \mathrm{cm} 2$ a un milímetro dedistancia (SDI radi plus, Australia). Sobre la superficie interna de la carilla, se aplicó ácido fluorhídrico al $9 \%$ (Porcelain etching, Ultradent-USA) durante 20 segundos; y, se procedió a lavar durante 40 segundos con spray de agua. Tras asegurarse de que la superficie esté seca, se aplicó silano (Ultradent-USA) durante un minuto e inmediatamente el agente cementante color traslúcido según el grupo, siguiendo las instrucciones de dosificación y mezcla establecidas por el fabricante. Los cementos usados según el grupo fueron los siguientes: G1 cemento RelyX ultimate (3m ESPEGermany), G 2 cemento RelyX Veneer ( $3 \mathrm{~m}$ ESPEGermany); G 3 resina fluida (Tetric-N-Flow, Ivoclar vivadent-Liechtensein, Europa). Posicionada la carilla, se realizó la eliminación de excesos y fotopolimerización mediante luz LED (SDI radi plus, Australia) durante 40 segundos, realizándose un nuevo registro de color mediante colorímetro Vita Easyshade (VitazahnFabrik- Alemania).

Los conjuntos "diente y carilla", fueron sometidos a 1200 ciclos de contacto con agua destilada a una temperatura de $\pm 5^{\circ}, \pm 37^{\circ}$ y \pm 55 durante 15 segundos (8). Tras lo cual, fue realizado un tercer registro del color en las condiciones descritas previamente. Los datos obtenidos de color fueron almacenados en tablas Excel previamente elaboradas, la diferencia en cuanto a tonos de color entre los tres periodos fue relacionada con los tres cementos empleados y analizados estadísticamente a través del programa STATA, considerándose qué valores de diferencia mayores indicaban mayor inestabilidad. 


\section{RESULTADOS}

El análisis descriptivo ejecutado, relacionando la primera y segunda evaluación, determinó que el RelyX ultimate (3m ESPE-Germany) presentó la menor variación de color $(6,17)$, mientras que el cemento RelyX Veneer (3m ESPE-Germany) y la resina fluida Tetric-N-Flow (Ivoclar vivadent-Liechtensein, Europa) mostraron una variación de 18,96 y 18,71 respectivamente. Al relacionar la segunda y tercera medición, el cemento RelyX ultimate (3m ESPEGermany) mostró mayor variación de color $(9,94)$ que los cementos RelyX Veneer (3m ESPE-Germany) y resina fluida Tetric-N-Flow, (Ivoclar vivadentLiechtensein, Europa), los cuales presentaron una media de cero (Figura 1 y Tabla 1).

Para afirmar que estas medias son significativamente diferentes al $5 \%$ de significancia y considerando su distribución normal en los grupos de cemento y diferencia, se aplica la prueba ANOVA con GamesHowell, puesto que no se asume homogeneidad en las varianzas y los grupos en análisis son equilibrados. Observando diferencia significativa entre casi todas las comparaciones, excepto en el cemento RelyX ultimate (3m ESPE-Germany) en sus periodos de medición. En el caso de los cementos RelyX Veneer (3m ESPEGermany) y resina fluida Tetric-N-Flow (Ivoclar vivadent-Liechtensein, Europa) la diferencia, considerando los periodos de medición, es significativa $(p=0,00)$ (Tabla 2), sin diferencia entre ellos en la

misma medición. Por lo tanto, el cemento $A$, dual RelyX ultimate (3m ESPE-Germany), muestra una mayor estabilidad en el color, pues sus diferencias entre $1-2$ y $2-3$ no son significativas.

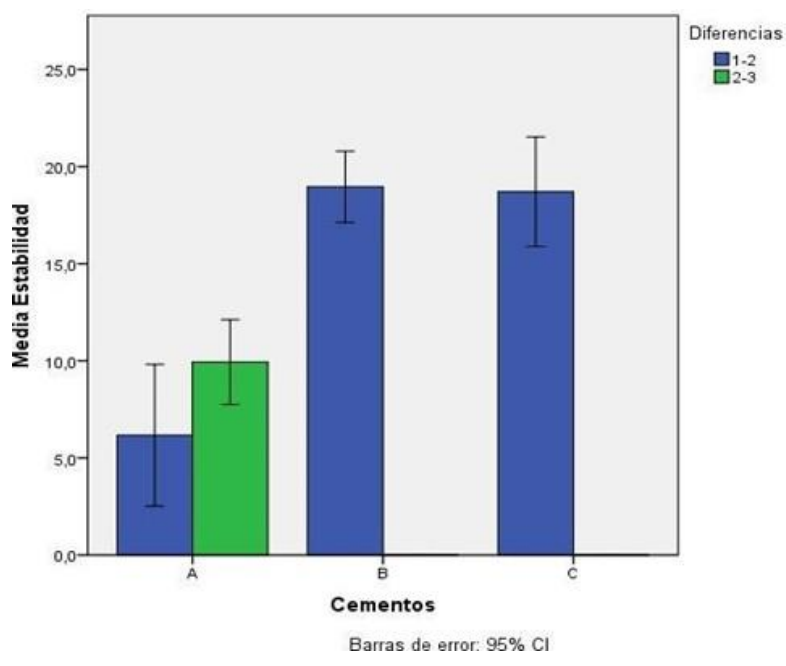

Figura 1. Descriptivos de la estabilidad del color de acuerdo a los cementos y diferencias aplicados.

Tabla 1. Descriptivos de la estabilidad del color de acuerdo a los cementos y diferencias aplicados.

\begin{tabular}{|c|c|c|c|c|c|c|c|c|}
\hline & \multirow{2}{*}{$\mathbf{N}$} & \multirow{2}{*}{ Media } & \multirow{2}{*}{$\begin{array}{l}\text { Desviación } \\
\text { típica }\end{array}$} & \multirow{2}{*}{$\begin{array}{l}\text { Error } \\
\text { típico }\end{array}$} & \multicolumn{2}{|c|}{$\begin{array}{l}\text { Intervalo de confianza para la } \\
\text { media al } 95 \%\end{array}$} & \multirow{2}{*}{ Mínimo } & \multirow{2}{*}{ Máximo } \\
\hline & & & & & Límite inferior & Límite superior & & \\
\hline A - Diferencia 1-2 & 10 & 6,17 & 5,10 & 1,61 & 2,52 & 9,82 & 0,10 & 14,10 \\
\hline A - Diferencia 2-3 & 10 & 9,94 & 3,05 & 0,97 & 7,76 & 12,12 & 3,80 & 13,20 \\
\hline B - Diferencia 1-2 & 10 & 18,96 & 2,56 & 0,81 & 17,13 & 20,79 & 13,70 & 21,80 \\
\hline B - Diferencia 2-3 & 10 & 0,00 & 0,00 & 0,00 & 0,00 & 0,00 & 0,00 & 0,00 \\
\hline C - Diferencia 1-2 & 10 & 18,71 & 3,95 & 1,25 & 15,89 & 21,53 & 10,60 & 24,60 \\
\hline C - Diferencia 2-3 & 10 & 0,00 & 0,00 & 0,00 & 0,00 & 0,00 & 0,00 & 0,00 \\
\hline Total & 60 & 8,96 & 8,40 & 1,08 & 6,79 & 11,13 & 0,00 & 24,60 \\
\hline
\end{tabular}

Tabla 2. ANOVA comparaciones múltiples

\begin{tabular}{cccc}
\hline & Tratamientos & Diferencia de medias & Significancia \\
\hline A - Diferencia 1-2 & B - Diferencia 1-2 & $-12,7900^{*}$ & 0,000 \\
& B - Diferencia 2-3 & $6,1700^{*}$ & 0,034 \\
& C - Diferencia 1-2 & $-12,5400^{*}$ & 0,000 \\
A - Diferencia 2-3 & C - Diferencia 2-3 & $6,1700^{*}$ & 0,034 \\
& B - Diferencia 1-2 & $-9,0200^{*}$ & 0,000 \\
& B - Diferencia 2-3 & $9,9400^{*}$ & 0,000 \\
B - Diferencia 1-2 & C - Diferencia 1-2 & $-8,7700^{*}$ & 0,000 \\
& C - Diferencia 2-3 & $9,9400^{*}$ & 0,000 \\
B - Diferencia 2-3 & B - Diferencia 2-3 & $18,9600^{*}$ & 0,000 \\
C - Diferencia 1-2 & C - Diferencia 2-3 & $18,9600^{*}$ & 0,000 \\
\hline
\end{tabular}




\section{DISCUSIÓN}

Los resultados obtenidos indican menor variación entre los dos periodos evaluados cuando se empleó el cemento dual RelyX ultimate ( $3 \mathrm{~m}$ ESPE-Germany), coincidiendo con estudios previos, donde se establece que los cambios de color son imperceptibles y clínicamente aceptables al emplear cementos de polimerización dual $(9,10)$, contrariamente a la teoría de que los agentes cementantes de curado dual producen más alteraciones de color $(11,12)$ inducidas por cambios químicos en el proceso de curado (13).

Fue evidente una estabilidad de color con un umbral aceptable en los tres materiales, incluso luego del envejecimiento al que fueron sometidos, lo que estaría asociado a la degradación de aminas residuales (14), componentes necesarios de los adhesivos para iniciar la polimerización, resultado de la temperatura y humedad (15). Es evidente la interacción de los materiales y la fuente lumínica como un factor capaz de influenciar en el proceso de excitación y reacción de los promotores de polimerización, que no sepresenta como elemento determinante en los sistemas de curado dual, donde gracias a una reacción química se concluye la polimerización ${ }^{(6)}$ sin presentar aminas aromáticas. Sin embargo, en las resinas fluidas, la estabilidad estaría asociada a la menor cantidad de BisGMA y la presencia de UDMA, que al reducir la cantidad de TEGMA presente en la matriz, reduce la reabsorción de agua, es decir, las uniones éter hidrófilas, reduciendo el cambio de color en el material (1). El uso de las cerámicas dentales es bien aceptado por su versatilidad mecánica y estabilidad biológica (16) en la que influyen directamente varios factores como (17), el color del diente ${ }^{(3)}$, grosor, tipo y tono de la cerámica ${ }^{(18)}$, junto con el tiempo de cocción y proporción del material, (19) sugiriendo, para minimizar estos, que el grosor de la pastilla cerámica para técnica prensada no sea menor de $2 \mathrm{~mm}$ (20)

Con la evolución de los protocolos adhesivos, los cementos resinosos han innovado de manera rápida y eficaz para conseguir un óptimo resultado al momento de utilizarlos en cualquier procedimiento clínico. Constituyen en la actualidad un excelente material que facilita el trabajo profesional brindando buenos resultados (19) con una unión eficaz alesmalte y la dentina asociada a los tratamientos previos y a su baja viscosidad (21), convirtiéndose en fundamental el respeto al protocolo adhesivo del cemento para lograr el éxito de una restauración y mantener estables sus propiedades ópticas ${ }^{(10)}$.

Los tres tipos de materiales resinosos empleados fueron translúcidos, considerando que el color de los materiales cementantes, constituye un factor muy importante en la estética. Por otro lado, sus marcas fueron escogidas tomando en cuenta su uso en el mercado ecuatoriano; sin embargo, otros materiales con las mismas características requieren ser evaluados. El método de evaluación mediante colorímetro Vita Easyshade (VitazahnFabrikAlemania) fue una limitante del estudio, por lo que el uso de espectrofotómetro constituiría una herramienta importante ${ }^{(18)}$ que merece ser empleada. Los medios de envejecimiento fueron similares a otros estudios; no obstante, se requieren evaluaciones futuras que contemplen tiempos más prolongados para poder guiar al clínico en el empleo de los materiales más adecuados ${ }^{(22) .}$

El envejecimiento artificial provocó cambio de color en todos los materiales cementantes evaluados, con menos variación entre los dos periodos evaluados en el cemento dual RelyX ultimate(3m ESPE-Germany). El cemento RelyX Veneer (3m ESPE-Germany) y la resina fluida Tetric-N-Flow, (Ivoclar vivadentLiechtensein, Europa) presentaron mayor inestabilidad del color con rangos plausibles clínicamente aceptables.

Contribuciones de autoría: DSM, JPRV, AA, EV, VBB y MF diseñaron el estudio, recopilaron y analizaron los datos, redactaron y aprobaron el manuscrito.

Fuente de financiamiento: Autofinanciado.

Conflicto de intereses: Los autores declararon no tener conflictos de interés.

\section{REFERENCIAS}

1. Radz GM. Minimum thickness anterior porcelain restorations. Dent Clin North Am. 2011; 55: 353370.

2 Prieto LT, Pimenta CT, Araujo JJ, Salles DC, Coppini EK, Sartini LA. Evaluation of degree of conversion and the effect of thermal aging on the color stability of resin cements and flowable composite. J Conserv Dent. 2018; 21 Suppl 1: 4751.

3. Alqahtani MQ, Aljurais RM, Alshaafi MM.The effects of different shades of resin luting cement on the color of ceramic veneers. Dent Mater J. 2012; 31 Suppl 3:354-361.

4. Dozic A, Voit NF, Zwartser R, Khashayar G, Aartman I. Color coverage of a newly developed system for color determination and reproduction in dentistry. J Prosthet Dent. 2010; 38: e50-e56.

5. Silami FD, Tonani R, Alandia CC, Pires-de- Souza, F. Influence of Different Types of Resin Luting Agents on Color Stability of Ceramic Laminate Veneers Subjected to Accelerated Artificial Aging. Braz Dent J. 2016; 27 Suppl 1:95- 100.

6. Almeida JR, Schmitt GU, Kaizer MR, Boscato N, Moraes RR. Resin-based luting agents and color stability of bonded ceramic veneers. J Prosthet Dent. 2015; 114 Suppl 2: 272-277. 
7. Bittencourt F. Barbosa B. Costa L. Atta T. Effect of the number of thermocycles on microleakage of resin composite restorations. Pesqui Odontol Bras. 2003; 17 suppl 4: 337-341.

8. Kelly JR, Nishimura I, Campbell SD. Ceramics in dentistry: historical roots and current perspectives. J Prosthet Dent. 1995; 75 Suppl 1, 18-32.

9. Bagis B, Turgut S. Optical properties of current ceramics systems for laminate veneers. Journal of dentistry. 2013; 41: e24-e30.

10. Ghavam M, Amani M, Saffarpour M. Effect of accelerated aging on the color and opacity of resin cements. Oper Dent. 2010; 3 Suppl 5: 605-609.

11. Nathanson D, Banasr F. Colour stability of resin cements--An in vitro study. Pract Proced Aesthet Dent. 2002; 14 Suppl 6: 449-455.

12 Noie F, O'Keefe KL, Powers JM. Colour stability of resin cements after accelerated aging. Int $\mathrm{J}$ Prosthodont. 1995; 8 Suppl 1:51-55.

13. Haralur SB, Alfaifi M, Almuaddi A, Al-Yazeedi M, AlAhmari A. The Effect of Accelerated Aging on the Colour Stability of Composite Resin Luting Cements using Different Bonding Techniques. J Clin Diagn Res. 2017; 11 Suppl 4: ZC57-ZC60.

14. Morita RK, Hayashida MF, Pupo YM, Berger G, Reggiani RD, Betiol EA. Minimally Invasive Laminate Veneers: Clinical Aspects in Treatment Planning and Cementation Procedures. Case Rep Dent. 2016;
1839793. http://doi.org/10.1155/2016/1839793

15. Turgut $S$, Bagis $B$. Colour stability of laminate veneers: an in vitro study. J Dent. 2011; 39: e57-e64.

16. Archegas LR, de Menezes Caldas DB, Rached RN, Soares P, Souza EM. Effect of ceramic veneer opacity and exposure time on the polymerization efficiency of resin cements. Oper Dent 2012; 37: 281-289.

17. Stevenson $B$, Ibbetson $R$. The effect of the substructure on the colour of samples/restorations veneered with ceramic: A literature review. J Dent. 2010; 38:361-368.

18. Ruyter IE, Nilner K, Moller B. Colour stability of dental composite resin materials for crown and bridge veneers. Dent Mater. 1987; 3 Suppl 5:246-51.

19. Dozić A, Kleverlaan CJ, Meegdes M, van der Zel J, Feilzer AJ. The influence of porcelain layer thickness on the final shade of ceramic restorations. J Prosthet Dent. 2003; 90: 563-570.

20. Peumans $M$, Van Meerbeek $B$, Lambrechts $P$, Vanherle G. Porcelain veneers: a review of the literature. $J$ Prosthet Dent. 2000; 28 Suppl 3: 163-177.

21. Kucukesmen HC, Usumez A, Ozturk N, Eroglu E. Change of shade by light polymerization in a resin cement polymerized beneath a ceramic restoration. $J$ Prosthet Dent. 2008; 36 suppl 3: 219-223.

22 Stewart PG, Jain P, Hodges J. Shear bond strength of resin cements to both ceramic and dentin, J Prosthet Dent. 2002; 88: 277-284. 